Supplement of

\title{
Improved representation of river runoff in Estimating the Circulation and Climate of the Ocean Version 4 (ECCOv4) simulations: implementation, evaluation, and impacts to coastal plume regions
}

Yang Feng et al.

Correspondence to: Yang Feng (yfeng1982@126.com)

The copyright of individual parts of the supplement might differ from the article licence. 


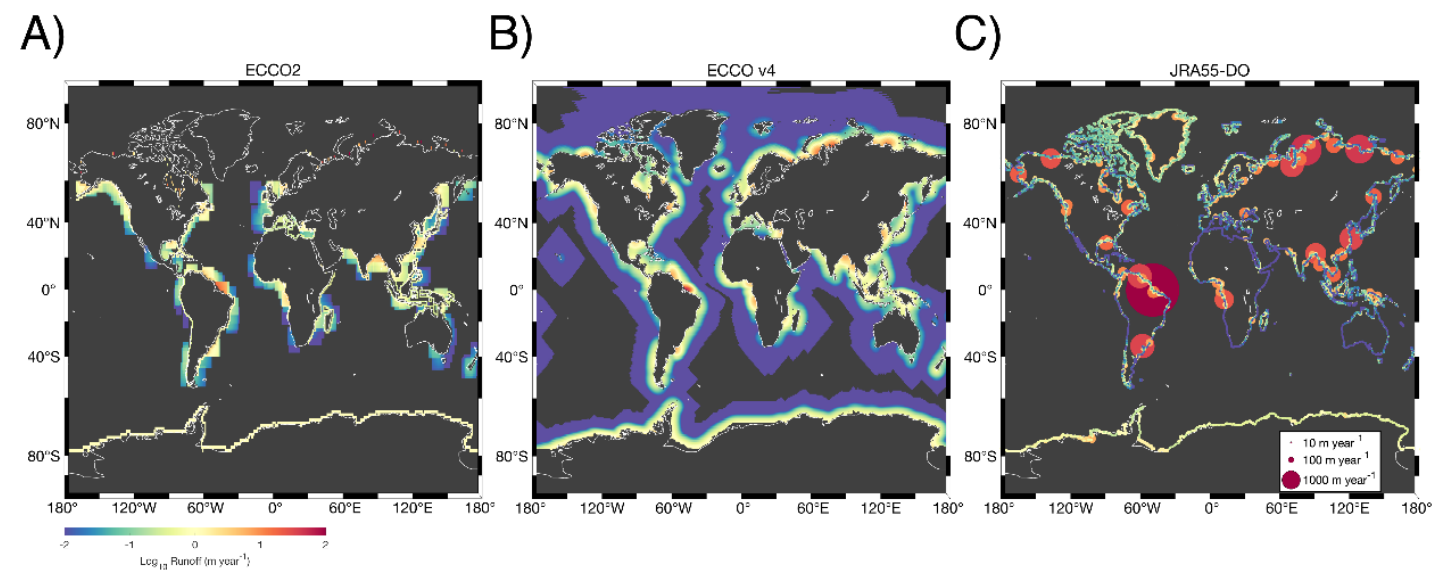

Figure S1: River discharge used in experiments: (a) $1^{\circ} \mathrm{x} 1^{\circ}$ climatological ECCO2 river forcing for CS510C; (b) $1^{\circ} \mathrm{X}$ $1^{\circ}$ climatological ECCOv4 river forcing for LLC90C and LLC270C; (c) the realistic JRA55-DO river forcing for LLC90R, 25 LLC270R, LLC540R and CS510R. 


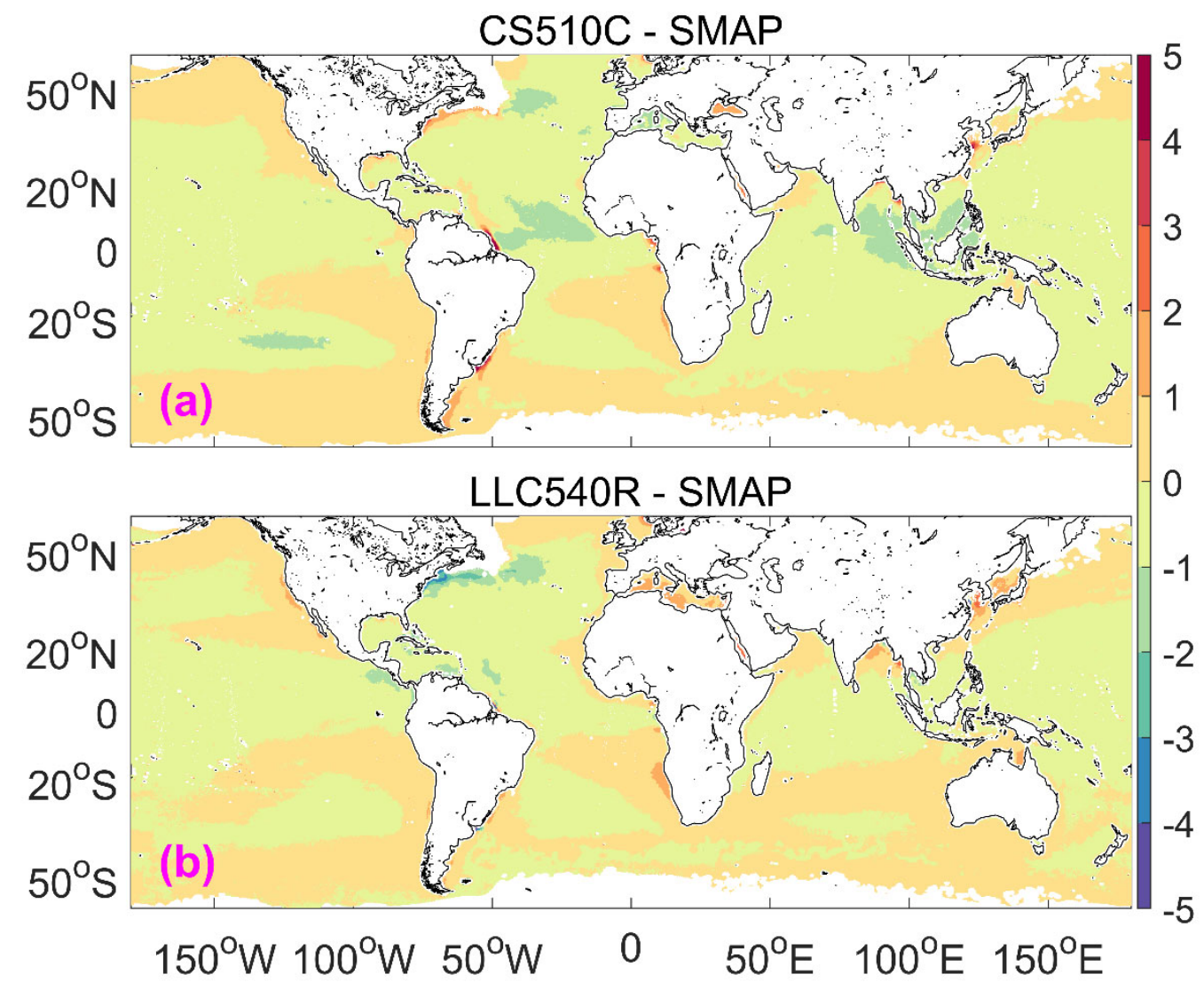

Figure S2: The 33-month (Apr 2015-Dec 2017) averaged salinity bias relative to SMAP for the global ocean for the reference run $(\mathrm{CS} 510 \mathrm{C})$ and highest resolution run with daily, point-source runoff forcing (LLC540R). The model SSS is interpolated to the $0.25^{\circ}$ SMAP grid for display purposes. 

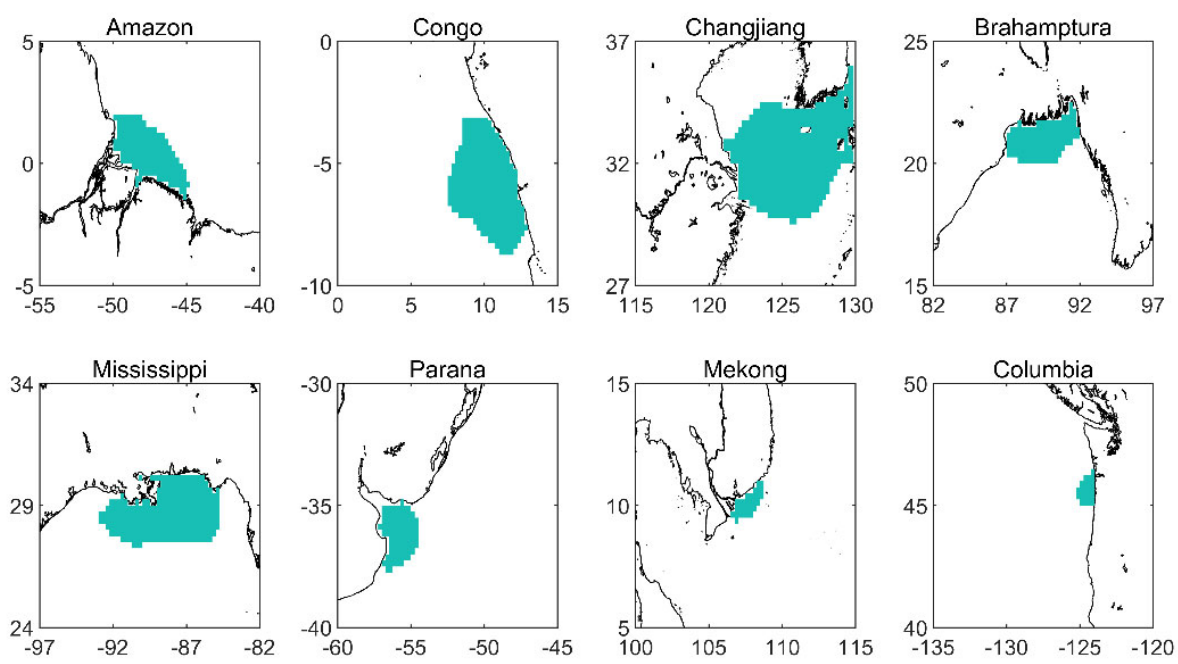

Figure S3: The eight river mouth regions that were identified by reconstructing the SSS anomaly field from the $1^{\text {st }}$ EOF mode of WOA18. 

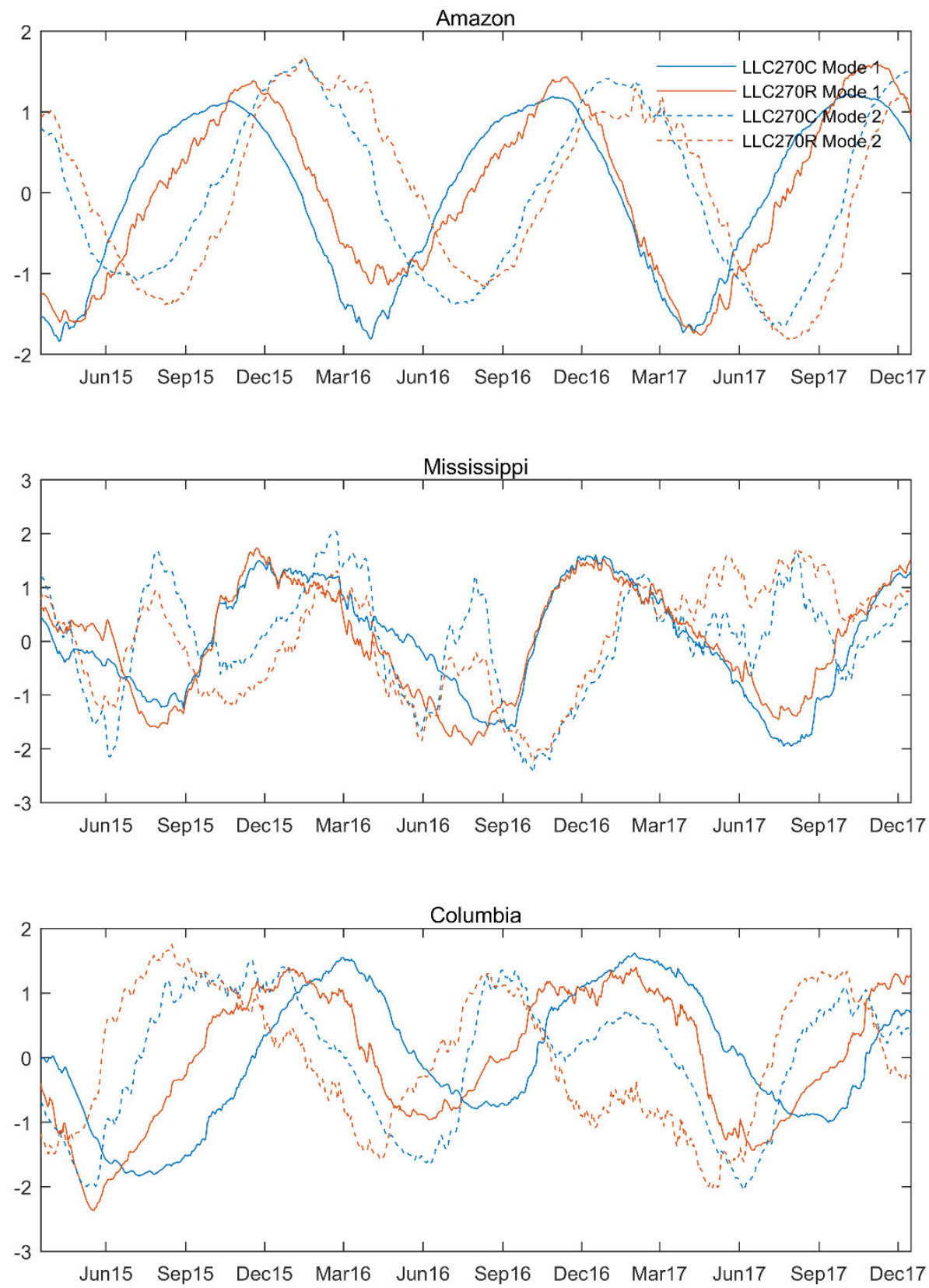

Figure S4: PC timeseries of the $1^{\text {st }}$ and $2^{\text {nd }}$ EOF of LLC270C and LLC270R simulations for the Amazon, Mississippi, and Columbia rivers. 

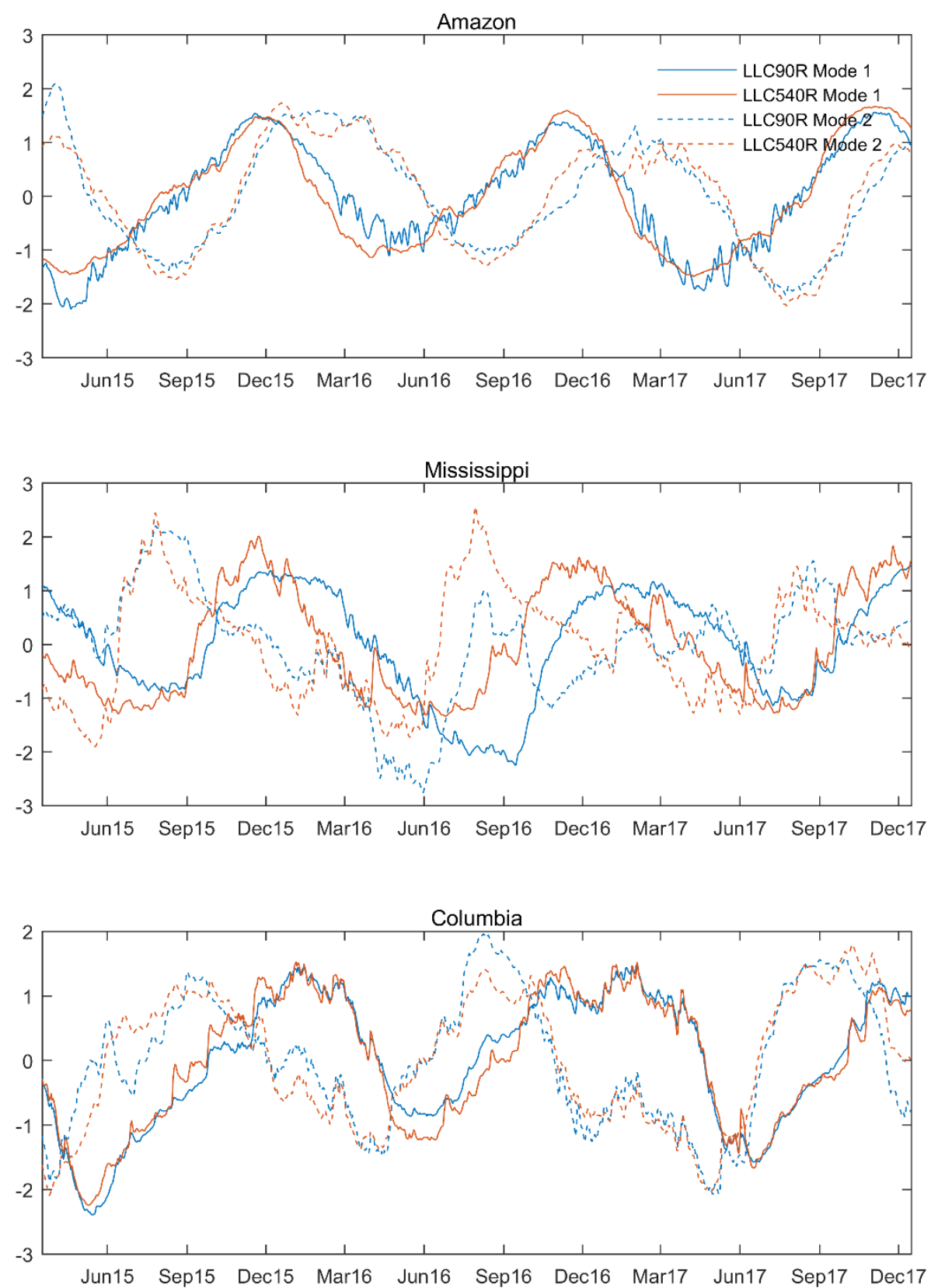

Figure S5: PC timeseries of the $1^{\text {st }}$ and $2^{\text {nd }}$ EOF of LLC90R and LLC540R simulations for the Amazon, Mississippi, and Columbia rivers. 

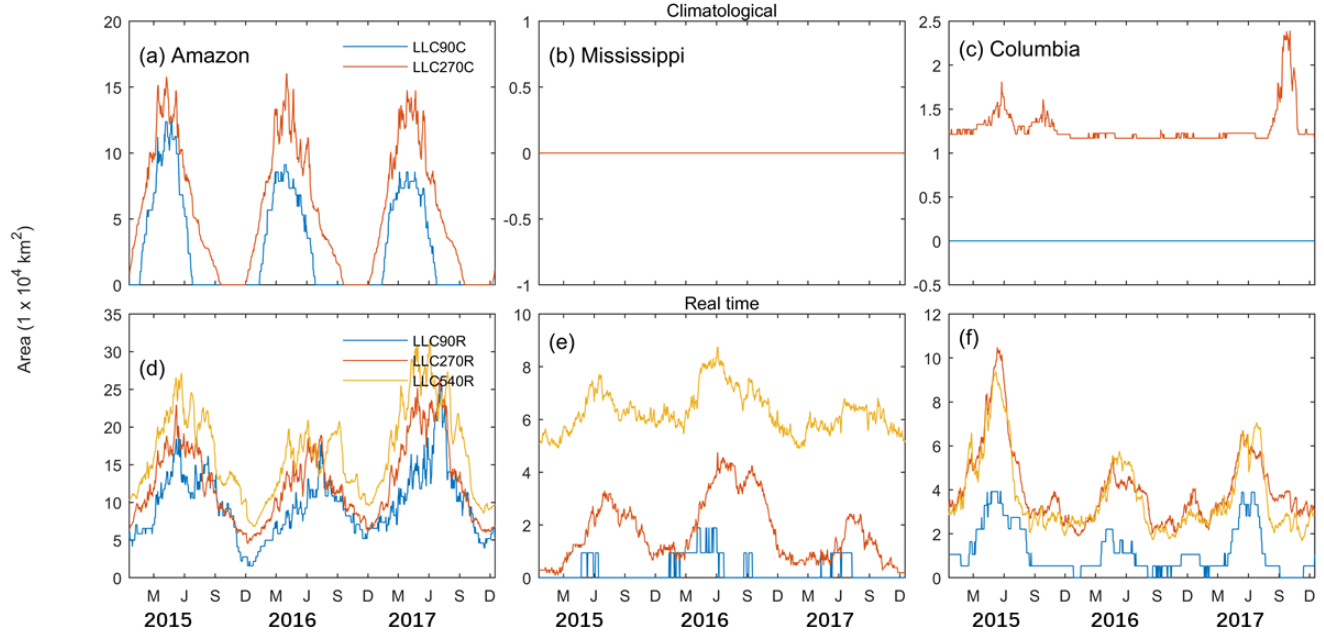

Figure S6: Area within 30 PSU in the Amazon, Mississippi, and Columbia river regions for the various experiments. 

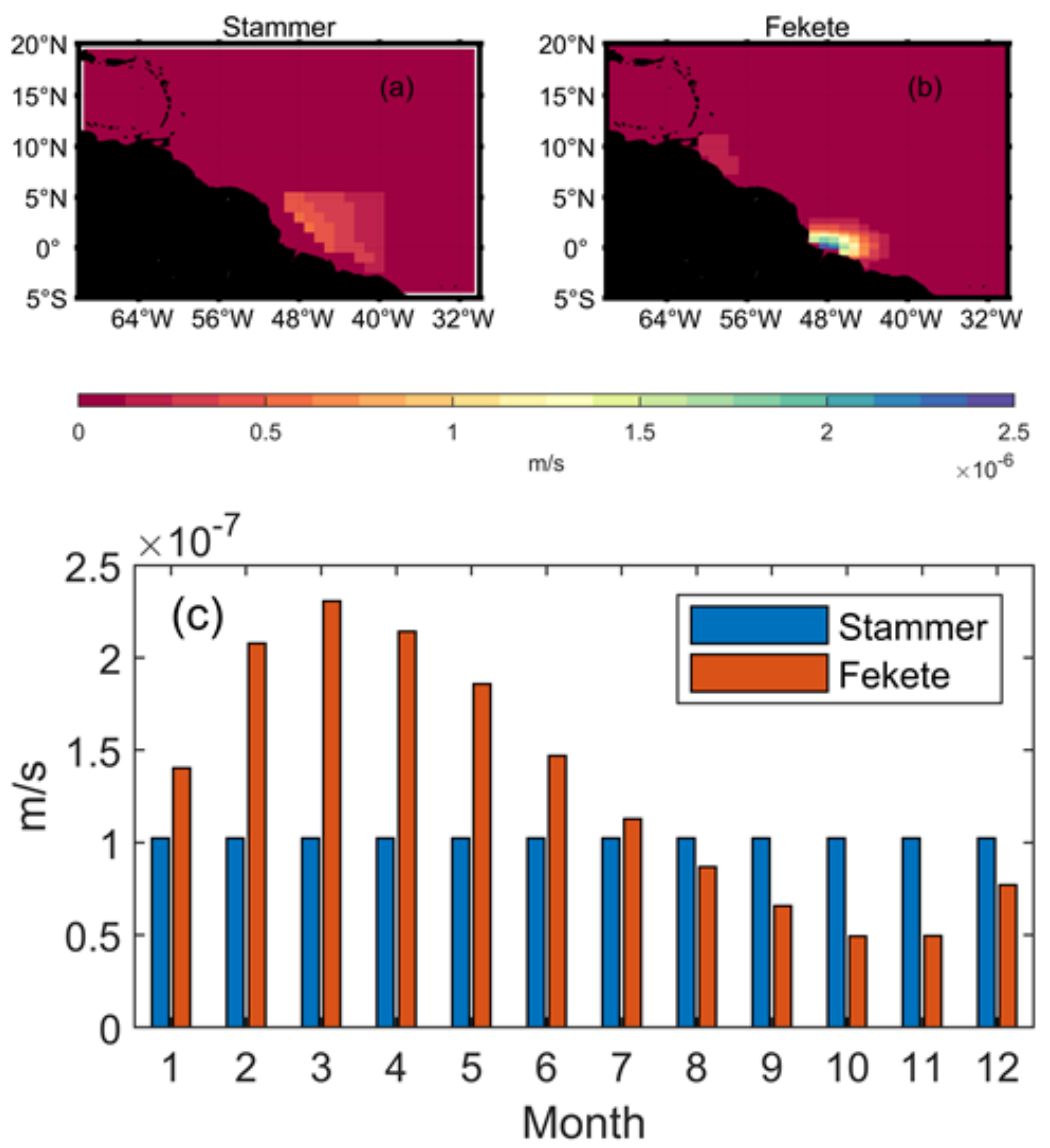

Figure S7: Comparison between ECCO2(Cube-sphere grid) Stammer and ECCOv4 (LLC grid) Fekete runoff forcing. 


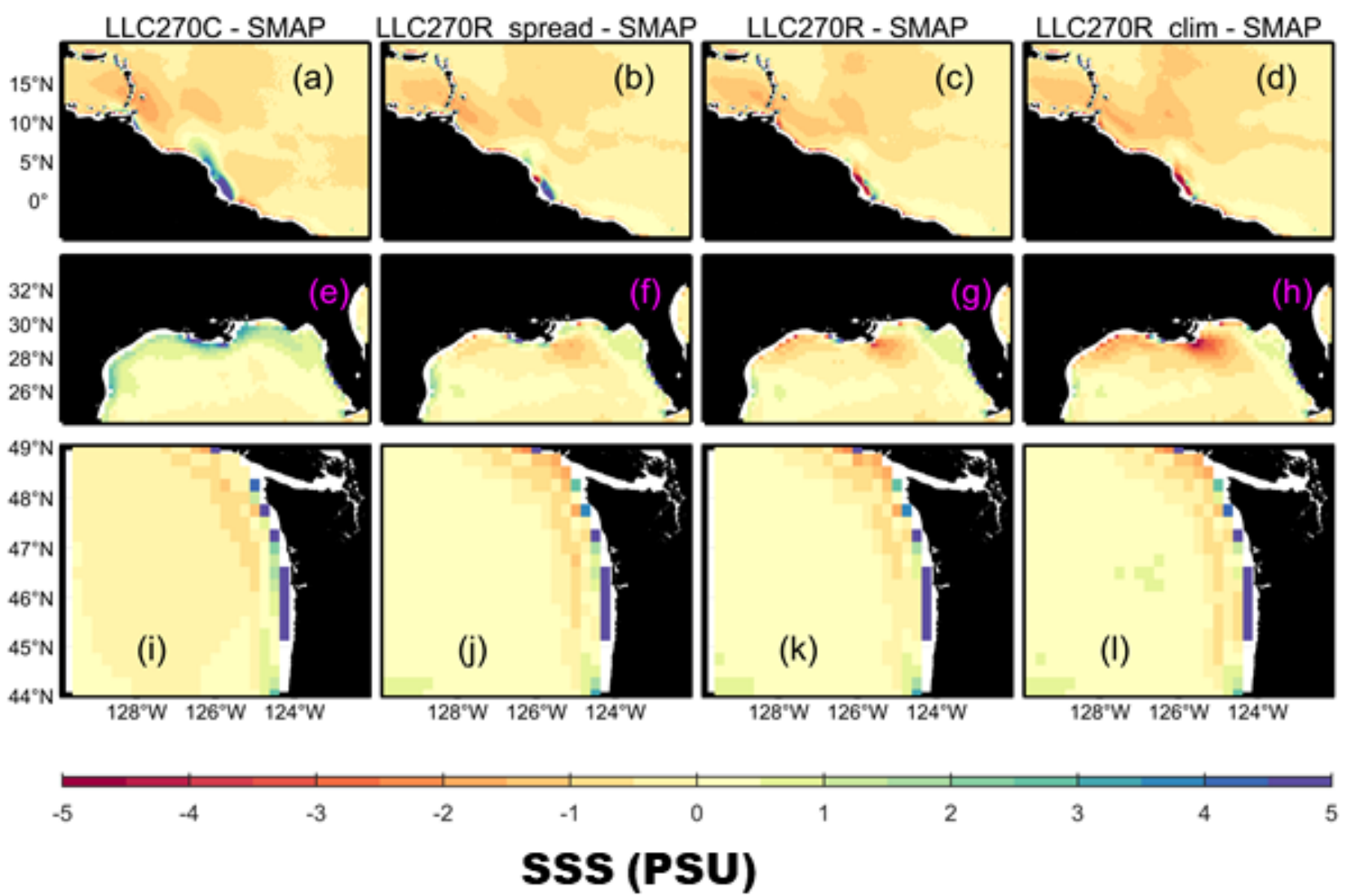

Figure S8: Zoomed-in view of SSS difference between different LLC270 experiments and SMAP observations for large (Amazon, a-d), medium (Mississippi, e-h), and small (Columbia, i-l) rivers. 